\title{
Tecnología, sociedad y comunicación. Hacia un modelo integrado de los efectos y determinaciones de las tecnologías de la comunicación ${ }^{*}$
}

\section{Luciano Elizalde}

Investigador del Consejo Nacional

de Investigaciones Científicas

y Técnicas (CONICET, Argentina)

\section{Resumen:}

El objetivo del trabajo es diseñar una hipótesis que integre la mayor cantidad de niveles de interdependencia entre la tecnología de la comunicación y la realidad social. Se parte de cuatro teorías que toman diferentes posiciones sobre la relación entre tecnología y sociedad: el "internalismo" y el "externalismo", por un lado, y el "determinismo tecnológico" y el "institucionalismo", por otro. Sobre la base de estas cuatro teorías, el estudio pone a prueba una nueva hipótesis -formada con sus argumentos más potentes y seguros- por medio del análisis de dos casos históricos: la imprenta y la televisión.

Palabras clave:

Tecnología - institución - comunicación de masas - estructura social.

\section{Abstract:}

The objective of the work is to design a hypothesis that integrates the greater amount of levels of interdependence between the technology of the communication and the social reality. Part of four theories that take different positions on the relation between technology and society: the "internalism" and the "exter-

* Agradezco las críticas y los consejos del Ing. Matías Munarris a una versión más simple de este estudio, aunque todos los errores y fallos son de mi absoluta responsabilidad, y al Lic. Diego Barceló por acercarme al trabajo de Pinch, Bijker y Hughes. 
nalism", on the one hand, and the "technological determinism" and the "institutionalism", on the other hand. On the base of these four theories, the study on approval puts a new hypothesis -formed with its more powerful and safe arguments- by means of the analysis of two historical cases: the press and the television.

Key words:

Technology, establishment, mass communication, social structure.

\section{Problemas y objetivos}

La sociología general y la sociología de la comunicación están interesadas, por distintos motivos, en explicar el proceso de desarrollo y de organización de la tecnología. La primera, a los fines de determinar qué es lo que modifica el desarrollo técnico y cuáles son las consecuencias de la tecnología sobre la sociedad (James Ellul, 1964: 149 y ss., pp. 248 y ss., pp. 319 y ss.; Merton, 1992: 648 y ss.; Giddens, 1997: 336-3357, pp. 655-656), mientras que la segunda pretende teorizar acerca de los procesos de producción, de distribución y de consumo de información, de textos o de representaciones en general que son afectados por las innovaciones tecnológicas (Bettetini, 1995: 19). Pero no es posible responder a la pregunta de cuáles son los efectos de las tecnologías sobre el proceso de comunicación, sin resolver antes la cuestión de las interdependencias entre técnica y sociedad. Por lo tanto, el problema que tratará de resolver este trabajo es doble y se encuentra resumido en dos tipos de interrogantes: por un lado, ¿cómo se organiza la técnica?, ¿de qué manera nace o se origina un determinado procedimiento tecnológico en cierto momento histórico?, ¿qué tipo de factores son de mayor importancia para el descubrimiento, invención, desarrollo, aplicación y adopción de una innovación tecnológica en la sociedad? Mientras que, desde el lado de la sociología de la comunicación, el problema tiene otro costado al que hay que responder: ¿cómo y de qué manera la técnica modifica el mundo social, psíquico y cultural del ser humano? ¿Cuáles y de qué orden o tipo de realidad son los procesos que producen cambios sobre la sociedad y la vida de los seres humanos? El primer tipo de interrogantes intenta ser respondido dentro de la discusión entre los "externalistas" y los "internalistas" del conocimiento y de la técnica. Los "internalistas" consideran que la técnica se determina sólo por mecanismos propios de la ciencia y de la misma técnica, dentro de los intereses y objetivos, motivos y teorías cientificas y técnicas. Los "externalistas", sin embargo, creen que la ciencia y la técnica están determinadas por aspectos sociales, políticos, económicos, culturales, psicológicos que no son inherentes al proceso técnico científico. Por otro lado, las preguntas orientadas hacia los efectos de la técnica sobre la sociedad o alguno de sus mecanismos (por ejemplo, la comunicación pública colectiva) se manifiesta y se expresa dentro de la discusión entre los "deterministas tecnológicos" (McLuhan, 1960, 1971, 1985a, 1985b, 1996; Ong, 1967a, 1967b, 1977, 1997) y los “institucionalistas" (Williams, 1971, 1975, 1982 1984, 1989). Los "deterministas tecno- 
lógicos" afirman que la sociedad y sus sistemas (economía, educación, política, cultura, familia, etcétera) experimentan cambios causados directamente por la tecnología, mientras que los "institucionalistas" rechazan esto y piensan que los efectos de las tecnologías están siempre mediados por procesos de institucionalización social, que son los derterminantes genuinos.

Expresado de un modo amplio, el problema que se pretende resolver está motivado en la falta de una respuesta integrada para las cuestiones que surgen de la relación entre la técnica y los procesos sociales. El objetivo, entonces, es desarrollar una hipótesis que explique el proceso de relación entre la técnica y la sociedad para ser aplicado a los medios de comunicación de masas y a las tecnologías digitalizadas de la comunicación y de la información.

Para la teoría de la comunicación de masas y, especialmente, para la sociología de la comunicación, ha sido central plantearse cuáles son los efectos de las tecnologías sobre los procesos de comunicación (privados y público, colectivos e interpersonales) es decir, de qué manera los mensajes, los procesos de codificación y de recepción y, en definitiva, todo proceso de comunicación (publicitario, periodístico, político, institucional) estaría o no determinado, y en qué grado o medida, por las tecnologías de la comunicación usadas. No hay comunicación sin técnica de producción, de distribución o de transmisión y de consumo de representaciones, de textos o de mensajes. En consecuencia, el problema de la tecnología ha sido central dentro de la teoría de la comunicación y de la teoría social aplicada a la comunicación de masas.

\subsection{Antecedentes teóricos: internalistas vs. externalistas y deterministas tecnológicos vs. institucionalistas}

Como antes se expuso, el problema general de la relación entre técnica y sociedad está marcado por dos discusiones diferentes. Por un lado, la discusión acerca de qué tipo de mecanismo produce o provoca cambios y transformaciones sobre la técnica y la ciencia aplicada. Por otro, el debate acerca de la medida o grado en que la técnica produce cambios directos en la sociedad (sobre la vida cotidiana, la economía, la educación, la cultura o la familia, en el caso de este trabajo, sobre la comunicación) o si esta técnica siempre e indefectiblemente se encuentra condicionada por las instituciones sociales que la ordenan y la organizan. A continuación se expondrán por separado un resumen de cada una de las dos discusiones a los fines de precisar los aportes que realizan a la solución acerca de la relación entre la técnica y la sociedad.

\subsubsection{Externalistas versus internalistas y el problema de los mecanismos de la técnica}

El problema que pretende resolver esta línea argumentativa de la discusión es si los cambios de la técnica son y/o deben ser impulsados, causados o motivados por aspectos inherentes a la técnica, a la ciencia 
y al conocimiento aplicado o si, por el contrario, las características y atributos, metas y objetivos de las tecnologías son determinados por aspectos externos o de naturaleza diferente a los de la ciencia y la técnica. En el fondo, el problema es saber si la sociedad es la que afecta el cambio tecnológico o si lo hace la propia organización científico-tecnológica.

La concepción cientificista y positivista de la ciencia y de la técnica denominada "internalista" (Bunge, 1998: 22) afirma que los cambios de la ciencia y de la técnica responden sólo a mecanismos propios o inherentes a estas áreas de conocimiento social. Para los "internalistas" la técnica es el resultado de las investigaciones precisas de los científicos o de los tecnócratas. La técnica cumple sólo y fundamentalmente las metas buscadas por quienes la crearon. Con lo cual, el proceso de creación, circulación y aplicación de una técnica es un proceso racional en cada una y en todas las etapas. Para la posición "internalista", la técnica está determinada, sobre todo, por procesos lógico-racionales que son coherentes para quienes crean y dan origen a una nueva técnica, para quienes la comercializan, la aplican o sólo la utilizan. Por lo general, desde este punto de vista, la técnica está definida por los objetivos de eficacia: la aparición y uso de una técnica responde sólo a criterios de eficacia racional. En esta dirección, entonces, se dirigen los internalistas al decir que tanto los procedimientos de invención, los descubrimientos, como la selección de los conocimientos cientificos y técnicos de generaciones anteriores de científicos están sólo y principalmente determinados por la propia racionalidad de la misma técnica. Cualquier otro factor externo a la técnica, a la ciencia y a los objetivos propios de la técnica y de la ciencia (por ejemplo, los hábitos de los usuarios) son poco importantes para la definición de ésta.

Del otro lado, la tesis de los autores que se identifican con la posición "externalista" es que las características de la técnica, sus usos y efectos están determinados por mecanismos no técnicos. En realidad, esta rama o línea de trabajo puede ser la "economía de la técnica", la "psicología de la técnica", la "sociología de la técnica" o la "antropología de la técnica". El modelo sociológico de la "masa crítica", por ejemplo, permite comprender de qué manera es posible que los cambios cuantitativos en la sociedad afecten a la tecnología. La explicación del funcionamiento, planificación, desarrollo y creación de la técnica están correlacionadas con la cantidad de usuarios que ésta comienza a tener a partir de cierto momento.

\subsubsection{Deterministas tecnológicos versus institucionalistas: el problema de los efectos} sobre la realidad social y sobre la comunicación

La segunda línea de discusión trata de los efectos de la técnica sobre la sociedad. El problema es determinar en qué medida las tecnologías son responsables de situaciones de bienestar o de penuria económica, social, cultural, psíquica, o si sólo son una base para que sobre ella se desarrollen los verdaderos mecanismos de cambios social: las instituciones económicas, culturales, religiosas, militares o científicas. Dentro del programa de investigación de los "deterministas tecnológicos" se encuentra la idea de que 
las técnicas modifican directamente las competencias corporales, psíquicas, sociales y culturales de los seres humanos. Esta es la idea de Harold Innis, Walter Ong, Marshal McLuhan en el ámbito de las teorías de los medios de comunicación y del cambio cultural. La posición mantenida en los años treinta por Theodor Adorno y Max Horkheimer en la Dialéctica de la Ilustración acepta la idea de que los procesos de industrialización son en sí mismos negativos para la formación de la sensibilidad estética y cultural de los seres humanos. Denis McQuail (1993: 135-137) afirma que el inicio de esta teoría se encuentra en los trabajos de Harold Innis, quien caracterizó a las civilizaciones de acuerdo con el tipo de comunicaciones que prevalecían. El cambio de un medio (de la piedra al papiro, por ejmplo) ocasionó el cambio del poder de los sacerdotes. En el resumen que Katz y Dayan (1995: 180-182) hacen de la teoría tecnológica de los efectos aparecen varios tipos diferentes de consencuencias de los medios técnicos: de la escritura, la lectura, la fotografía, el cine, televisión, etc. Una versión actual de esta teoría la continúa defendiendo el politicólogo Giovanni Sartori (1992: 305-316) al afirmar que la televisión y la imagen, en general, han destituido a los textos escritos y al hábito de la lectura, con lo cual se está realizando un cambios antropológico en el ser humano, cambio que va desde el homo sapiens al homo videns. Finalmente, una versión mesurada, aunque determinista especialmente desde el punto de vista de las tecnologías de la comunicación, es la de Niklas Luhmann (Luhmann y De Georgi, 1993) al proponer una hipótesis explicativa de la evolución de las sociedades humanas teniendo en cuenta los cambios antropológicos de los medios de comunicación (escritura, imprenta, medios de difusión, nuevos medios).

Al otro lado de esta teoría se encuentran los autores que consideran que las técnicas no pueden afectar a los seres humanos sin la mediación de la organización institucional. Para ellos, son las instituciones y los elementos que las constituyen los que modifican negativa o positivamente a los seres humanos, pero no las tecnologías por sí solas. Raymond Williams es uno de los representantes más contundentes de esta posición. Considera que es un error concluir que la técnica es la causa de las diferencias sociales. En América Latina, Jesús Martín Barbero (1991: 56 y ss.) comparte la concepción de Williams de que las "mediaciones" institucionales que se interponen entre las técnicas y los cambios sociales. En realidad, es siempre lo social o lo cultural lo que activa y produce el cambio.

\subsection{Metodología}

El método utilizado para llegar a los resultados del trabajo es histórico social, aunque con el agregado del método hipotético-deductivo. En primer lugar, se parte del marco de discusión teórico constituido por las principales líneas de discusión del tema dentro de la sociología general, la sociología de la técnica y la sociología de la comunicación. Segundo, se aíslan los argumentos e hipótesis que permiten explicar cada una de las lagunas del problema. Y tercero, se utiliza la historia de la tecnología y, especialmente, de la tecnología de la comunicación, para aplicar el modelo teórico y analizar los resultados. 


\section{Hipótesis sobre la relación técnica-sociedad}

\subsection{Hipótesis}

El resumen de la hipótesis consta de tres puntos importantes. En primer lugar, es necesario reconocer que la tecnología -como actividad humana- cuenta con cierta autonomía relativa. Esto significa que se encuentra determinada -aunque no exclusivamente- por procesos sociales de naturaleza externa a la técnica. Es decir, la tecnología es parte de la sociedad, es el resultado de procesos sociales no tecnológicos. En segundo lugar, la tecnología como actividad y realidad con autonomía relativa, genera efectos y determinaciones sobre la realidad social, es decir, modifica aspectos culturales, económicos, políticos, militares, educativos, familiares, laborales, burocráticos, etcétera. Finalmente, los procesos de comunicación colectivos están determinados por la interacción entre tecnología y sociedad.

Todo esto significa que la tecnología no sólo es el resultado de mecanismos y de decisiones estrictamente técnico-científicas, sino que recibe efectos de procesos sociales externos al campo científico tecnológico. Sin embargo, esto no significa que la técnica no cuente con el grado de autonomía necesario y suficiente como para que ciertos cambios y transformaciones se realicen con independencia de los mecanismos sociales. Por otro lado, la tecnología provoca cambios en la realidad humana. Cambios y transformaciones que no sólo se realizan por medio de mecanismos sociales históricamente particularizados, como los institucionalistas pretenden que sea, sino que se desarrollan cambios y estabilizaciones de cambios influyendo sobre mecanismos biológicos universales. Esta última afirmación corresponde a los deterministas tecnológicos. Por lo tanto, la hipótesis afirma que la relación entre la realidad tecnológica y realidad social contiene varios niveles diferentes de organización y de formación. Los mecanismos sociales determinantes de la tecnología y los mecanismos tecnológicos determinantes de lo social se encuentran entrelazados y funcionan todos y cada uno, pero en diferente nivel de realidad. La técnica recibe determinaciones de la vida social en la que se desarrolla, pero las sociedades también son cambiadas y transformadas por patrones de comportamiento definidos por mecanismos de la técnica utilizada por grupos de personas, con o sin patrones organizativos. Entonces, el desarrollo de las relaciones entre técnica y sociedad se produce dentro de un contexto de complejidad que implica varios tipos diferentes de mecanismos estrictamente técnicos y otros propiamente sociales. "Complejidad" significa la intervención de más de dos niveles de organización de realidad en un mismo fenómeno (por ejemplo, biológico, psíquico y social; o técnico, psicológico y social).

\subsection{Proposiciones de la hipótesis}

Los juicios más importantes y que resumen la idea anterior son los siguientes:

P1. Es necesario, aunque no es suficiente, obtener conocimiento - científico o pre-cientificos-para la invención y el desarrollo de un nuevo artefacto técnico que tenga la capacidad de solucionar 
mejor, en términos relativos, un viejo problema. Desde este punto de vista, la técnica es una herramienta o un instrumento, es decir, algo que se utiliza para la solución de un problema o la consecución de un fin, por lo tanto, está determinada por aspectos específicos relacionados con los avances e innovaciones que hacen más eficaz llegar a un objetivo. Sin embargo, esta posición internalista no es suficiente ya que, para el uso y la aplicación efectiva de una técnica no son suficientes sólo mecanismos técnico cognoscitivos.

P2. Sólo es posible resolver un problema con un nuevo artefacto técnico, si éste es aceptado por diferentes grupos sociales que lo utilizarán para fines que no habian sido pensados ni planificados por sus inventores. El instrumento técnico es recibido de un modo subjetivo, es decir, particular, por diferentes grupos y por personas individuales; de hecho, estos grupos (profesionales, ideológicos, religiosos, de edad, económicos, etc.) pueden producir cambios en los usos y en las valoraciones en relación con la definición original del instrumento y con el intento de institucionalización inicial, que pretende ser universal y general.

P3. El proceso de discusión, de disensos, de litigios y de conflictos entre grupos sociales diferentes por la definición, desarrollo y/o aplicación del nuevo artefacto, se cierra con el proceso de institucionalización o de estabilización. Un instrumento técnico cualquiera, después de ser ordenado bajo criterios y principios técnicos de eficacia, es organizado por un proceso de institucionalización, es decir, de normativización, de jerarquización social, de significación cultural para que esta nueva técnica pueda ser adoptada en una sociedad.

P4. Finalmente, si y sólo si un artefacto técnico pasa por este proceso de institucionalización es posible que desarrolle y provoque ciertos efectos corporales, biológicos, de orden universal en quienes usan la técnica. A largo plazo, o sea, dentro de períodos de tiempo dentro de los que la técnica en cuestión ha alcanzado un uso intensivo bajo una o más de una forma institucional, los usuarios pueden experimentar cambios en el nivel corporal, psíquico y social; estos cambios pueden ser, tanto, efectos particularizados por las diferencias institucionales y grupales, por un lado, o efectos universalizados por la relación entre las características corporales del género humano y las modificaciones técnicas incorporadas por el instrumento utilizado.

\section{Desarrollo de una teoría multidimensional de la técnica y la sociedad}

A continuación se desarrollarán las cuatro proposiciones que se han expuesto resumidamente en el apartado 2 .

\subsection{Desarrollo de la proposición 1}

La técnica como acción instrumental resuelve un tipo de problemas y/o cumple ciertos fines. Aunque esto sólo es necesario pero no suficiente para que de hecho cumpla con los objetivos fijados por técnicos 
que la diseñaron. Esto significa que una técnica sirve para hacer algo. Debe resolver algún problema con cierto grado de eficacia. En el caso de la técnica o tecnología de la comunicación, sus innovaciones deberían prestar un servicio o deberían mejorar el rendimiento en la producción, la transmisión, el almacenamiento y la recuperación de representaciones cognoscitivas (Bettetini, 1995: 29 y ss.). Las técnicas que mejoran la comunicación han permitido un proceso más eficaz -en sentido integral o global, es decir, económico, moral, cultural, religioso, estatal, familiar, etcétera- para producir de un modo inteligible y sin errores un texto o representación, para transmitirlo trascendiendo el modo de organizar el tiempo y el espacio que eran normales hasta ese momento; han tenido que mejorar los modos de utilización de la técnica, de facilitarla corporal y psicológicamente. Además, han mejorado el proceso de gestión del conocimiento práctico, es decir, han mejorado la manera de copiar, de manipular, de almacenar y de recuperar conocimiento. Dice Norbert Elias que las técnicas realizan un proceso de mejoramiento relativo si se compara la manera de resolver el mismo problema antes y después de la utilización de la técnica (Elias, 1998: 455). Elias dice que las técnicas se aplican porque traen aparejados "mejores condiciones de vida". No son "buenas condiciones de vida", sino mejores, en tanto se realiza una comparación efectiva entre un contexto y otro de resolución del problema: antes y después de ser aplicada la técnica.

La experimentación y el desarrollo de nuevas técnicas puede diferenciarse entre las que fueron motivadas por un conocimiento no científico -antes del proceso de institucionalización de la ciencia moderna-y las que son el resultado de los avances y de las conclusiones del conocimiento de la ciencia. La tendencia actual es definir la técnica como una aplicación de la ciencia, sea para atacarla como para defenderla. Mario Bunge diferencia entre "técnicas tradicionales" y "técnicas científicas" (Bunge, 1991: 27). La ciencia, dice Bunge, precede a la praxis dándole herramientas y artefactos. Una serie importante de técnicas (químicas, biotecnologías, psicotécnicas, electrónica, etc.) se inspiraron en las ciencias básicas. Y esto es muy positivo. Dice Bunge: “(...) todo diseño técnico moderno utiliza conocimientos científicos y, antes de poder aplicar un conocimiento, es preciso haberlo producido o tomado prestado" (Bunge, 1991: 27). En una definición más estricta, Bunge agrega que la tecnología tiene dos características: primero, debe ser comparable y controlada por la ciencia y el método científico y segundo, se la emplea para "controlar, transformar o crear cosas o productos, naturales o sociales" (Bunge, 1980: 190). Sin embargo, sería posible que alguien con ingenio especial, con mucha creatividad en relación con sus contemporáneos, pero sin formación científica, formule el diseño de un artefacto que mejore la manera relativa de solucionar un problema. Ahora bien, tiene razón Bunge cuando dice que, históricamente, la ciencia ha precedido a la técnica en el último siglo, aunque esto no debería significar que no puedan darse desarrollos de técnicas con una base no científica o sin el proceso de planificación del método científico. Por otro lado, Habermas (1989: 73-74), siguiendo directamente Marcuse e indirectamente a otros miembros de la Escuela de Frankfurt, considera que la racionalización ha sido adquirida de tal modo que terminado siendo "naturalizada" por la sociedad moderna en la forma "acción racional con 
arreglo a fines" de acuerdo con Max Weber. La naturalización o la normalización de este tipo de racionalidad -sumado al hecho de que esta definición de racionalidad monopoliza la idea misma de racionalidad en Occidente- se transforma en "ideología", es decir, en conocimiento para el dominio y no para la verdad. Con lo cual, la técnica es considerada un elemento ideológico en el mundo moderno si se lo define como el resultado de la ciencia. Esta concepción proviene de Max Weber. Para él la técnica está organizada dentro del marco de las "acciones racionales con arreglo a fines". Y desde el punto de vista de la antropología filosófica de Arnold Gehlen (1980: 56), la técnica es el resultado de lo "faltante" en el ser humano. Sobre la base de una definición de Nietzsche, Gehlen afirma que el hombre es un ser inacabado, no terminado, desvalido, con lo cual necesita de la ayuda que la técnica le provee. Se podría decir, a partir de Weber y también de Gehlen, que la técnica es una continuación, un perfeccionamiento de la acción humana ${ }^{1}$.

La esencia de la definición de técnica, entonces, está contenida en la definición de Max Weber: el conjunto de medios aplicados a una actividad, que se diferencian de la finalidad o fin al que se oriente (Weber, 1996: 47 y ss.). La técnica es el proceso de selección de los mejores medios, los más racionales, para alcanzar un fin, casi siempre problemático o no alcanzado antes con otros procedimientos menores adecuados o racionales.

\section{2. $\quad$ Desarrollo de la proposición 2}

La técnica en tanto un instrumento es interpretado de un modo subjetivo por grupos diferentes de usuarios. El hecho de que un cierto procedimiento técnico diseñado y puesto en funcionamiento por los expertos en el laboratorio, probado y vuelto a probar, sólo constituye el comienzo o la primera fase del desarrollo, aplicación y uso del artefacto. Las técnicas y sus inventores, más allá de la racionalidad del procedimiento, se encuentran con que éstas serán usadas dentro de ciertos contextos sociales y culturales. Aquí aparece con cierta fuerza la tesis del constructivismo social (Pinch y Bijker, 1984). El constructivismo social, como posición externalista extrema, postula que las técnicas y los artefactos son el resultado de un proceso social y cultural. No es posible explicar el desarrollo de la tecnología como si la ciencia y la técnica fuesen una estructura monolítica y cerrada. En cambio, afirman que la ciencia y la técnica son construcciones socioculturales, que definen sus objetivos y propósitos dentro de la cultura. No se puede tratar a la relación entre técnica y tecnología de un modo unidireccional. Por el contrario, se realiza un proceso de negociación social y de representación de distinciones (Pinch y Bijker, 1984: 403-404). En realidad, si se modera el argumento, el constructivismo posibilita explicar una instancia del desarrollo de la tecnología que no lo puede explicar el "internalismo" con la teoría del proceso

${ }^{1}$ En esto coinciden con Aristóteles: la acción para éste es siempre de tipo teleológica, por lo tanto, persigue un fin o meta. Toda acción tiene un fin y los seres humanos mejoran los medios técnicos para alcanzarlos. 
de innovación. Ésta considera que el formato del proceso de la tecnología en la sociedad es el siguiente según la exposición de Pinch y Bijker (1984: 405):

\section{Investigación básica}

Investigación
aplicada

\section{Desarrollo} tecnológico

\section{Producción}

Uso

En cambio, el proceso identificado por el constructivismo (Pinch y Bijker, 1984: 412) es otro:

\section{Artefacto}

\section{Grupo social}

\section{Problema}

\section{Solución}

El artefacto producido y desarrollo por científicos y tecnócratas -o sólo por aficionados muy informados en sociedades menos científicas- no será usado de un modo definitivo hasta que no sea manipulado por diferentes grupos sociales, con intereses específicos y generales distintos entre sí y con formas de vida que no son comunes. Pinch y Bijker (1984: 414) afirman que un "relevant social group" puede concentrar ciertos requirimientos y demandas, nuevos significados y usos para cierto artefacto. Por lo tanto, el artefacto no podrá tener un funcionamiento plenamente eficaz hasta que estas diferencias no hayan sido resueltas por el técnico, sea el inventor o el dueño de la patente. Las diferencias interpretativas y retóricas que los grupos relevantes le dan al artefacto, lleva a que los investigadores o fabricantes deban realizar cambios. Estos cambios, entre otros, pueden ser orientados a que el artefacto solucione otros problemas que no se habían tenido en cuenta hasta el momento que el grupo tomara contacto y aplicara la técnica en cuestión.

Diferentes grupos culturales, con distintas costumbres, hábitos, usos, con creencias compatibles o incompatibles en relación con la tecnología, modifican el artefacto técnico. El mundo de la diferencia de los hábitos humanos no es un muro infranqueable, pero puede ser lo suficientemente denso como para que la mejor técnica quede sin ser usada hasta que se desempeñen cambios en los patrones sociales. Las expectativas que los expertos tienen sobre el modo en que se recibirá una cierta técnica es normal que no coincida, por lo menos en un principio, con las expectativas de los legos en relación con los alcances y cambios que los medios o procedimientos de la técnica imponen a sus modos de hacer, de pensar y de ser. Cada técnica será adaptada a las necesidades particulares de ciertos grupos sociales, a sus creencias, expectativas y valores. Pinch y Bijker (1984: 416) hacen referencia a diferentes conflictos que plantean los grupos sociales (seguridad para el uso, derechos de uso, discriminación, efectos morales, etc.) y a la estabilización de esos conflictos, es decir, a la realización de un equilibrio entre los grupos sociales que intervienen en el proceso de definición y el uso de la técnica en un proceso de institucionalización.

Algunas veces, el cambio necesario y suficiente es la transformación generacional que hace posible que la técnica -hasta ese momento rechazada- sea usada por una mayor cantidad de personas. El proceso de aumento de usuarios ha sido bastante estudiado por el modelo de la masa crítica (Oliver y Marwell, 2001: 300-302; Williams, Strover y Grant, 1996: 624; Schelling, 1978: 91-110). El hecho de que 
se produzca un aumento de usuarios de un artefacto técnico, genera -en alguna de las fases del proceso de aumento- un cambio de orden cualitativo que tiene como consecuencia una transformación más amplia, incluyendo a otros grupos de usuarios hasta el momento indiferente o con actitudes negativas en relación con esta tecnología. El aumento de usuarios de una técnica modifica las relaciones de interdependencia entre el grupo de usuarios y de no usuarios. Los no usuarios perciben que las relaciones e interacciones con otras personas sociales -que son usuarios-son más fáciles si se tiene la tecnología; también es posible que el aumento de usuarios genere un efecto demostración que afecte el respeto social y la reputación por la técnica que se encuentra en vías de adopción. No tener la técnica pasa a ser algo extraño, y humillante para muchas personas sociales.

En resumen, la técnica tiene capacidad de afectar o de determinar el comportamiento de un grupo de personas si es aceptada para ser aplicada con ciertos fines. La aplicación de la técnica para hacer algo de un modo más eficaz puede ser modificada por los gustos, necesidades, intereses, hábitos y valores de un grupo social. Este obstáculo con el que se encuentra la técnica como herramienta o instrumento, permite que la realidad social influya sobre la técnica gracias a las aplicaciones no pensadas y no queridas por los inventores o los expertos.

\section{3. $\quad$ Desarrollo de la proposición 3}

Un instrumento técnico que es afectado por diferentes definiciones sociales adquiere una forma institucional objetiva, es decir, una definición social menos cambiable por otras interpretaciones.

La fase de la institucionalización de una tecnología es denominada por Pinch y Bijker, proceso de clausura y estabilización (Pinch y Bijker, 1984: 424 y ss; Bijker, 1993: 121). Es el mismo proceso social explicado por Berger y Luckmann (1989: 25 y ss.; 47-48), por John Searle (1997: 16 y ss.), por Mary Douglas (1993: 27 y ss.), por Helmut Schelsky (1967a: 17-18; 1967b: 59 y ss.) o por Norbert Elias (1998). En general, todos estos autores tienen en común el hecho de que afirman que la técnica -u otro objeto social-se institucionaliza en el momento en que se objetivan ciertas normas sociales, leyes, expectativas, significados y valores asociados y adheridos por el hábito a un cierto artefacto técnico. De un modo más específico, Raymond Williams (1989: 152-153) considera que sólo es posible pensar y explicar la relación entre técnica y sociedad dentro de la sociología de las instituciones. Considera que el determinismo tecnológico se equivoca al creer que los seres humanos se adaptan a nuevas tecnologías de un modo directo. Cualquier estudio técnico o científico, dice Williams, se realiza en un marco de relaciones sociales y formas culturales ya existentes. Además, las ideas y las aplicaciones de la ciencia quedan atrapadas dentro de ciertas formas sociales que terminan definiendo la auténtica e integral utilización de la técnica en cuestión.

De acuerdo con la postura "institucionalista", la técnica no tiene, por sí misma, la capacidad de modificar la vida social de los seres humanos. Los institucionalistas creen que no es verdad que un cierto 
tipo de técnica cambia, para mal o para bien, la vida de los seres humanos. Por el contrario, creen que las tecnologías son organizadas dentro de una serie de reglas sociales, formales e informales, caracterizadas por ciertos valores, significados, atributos simbólicos de mayor o menor reputación, que es lo que definitivamente determinan el mundo social humano. Las diferentes posturas de los institucionalistas tienen en común que consideran que los determinantes centrales de la vida humana son entidades sociales objetivas que presionan y coercionan a las personas sociales por medio de mecanismos de internalización (autocoacción) y de imitación social. Para que una institución sea real, no puede desaparecer a voluntad de una conciencia sino que permanece objetiva ante ella. Para que suceda, es necesario un proceso que comienza con la tipificación y habituación de comportamientos, pasa por la vinculación con ciertos significados y procedimientos simbólicos hasta la aceptación colectiva de sus intenciones y regulaciones (Berger y Luckmann, 1989: 67 y ss.; Douglas, 1993: 29; Schelsky, 1967b: 57 y ss.; Searle, 1997: 35 y ss.).

El hecho de que una técnica sea organizada por medio de ciertas reglas y valores, es decir, que los grupos de organizadores le concedan ciertas formas políticas, económicas, sociales, culturales, legales, etcétera, hace que la técnica produzca ciertos tipos de efectos. Es la determinación social más fuerte que recibe un instrumento técnico.

\subsection{Desarrollo de la proposición 4}

Las diferencias sociales de interpretación y de institucionalización que la sociedad instaura sobre la técnica no pueden restringir los efectos generales o universales que el instrumento o artefacto técnico tiene capacidad de desarrollar sobre la dimensión corporal de los seres humanos una vez que se forma un ambiente o entorno mediático o tecnológico alrededor de las personas sociales. Solamente aquellos artefactos técnicos que resuelven eficazmente una gama de problemas de la vida humana, que han sido aceptados para ciertos usos por determinados grupos sociales (de profesionales, ideológicos, religiosos, de hábitos de vida, etc.) y que han sido organizados institucionalmente (en una economía de mercado o bajo protección estatal, por ejemplo), se encuentra en capacidad de desarrollar una serie de efectos más o menos aplanadores y universales sobre el cuerpo y los sentidos, el tiempo y el espacio, la psiquis y las relaciones sociales de los usuarios. Es decir, después de que el artefacto es definido por un proceso de determinación técnica y social, está en capacidad para conformar un entorno o ambiente en el que aparecen los efectos "técnicos" sobre los seres humanos (Mc Luhan, 1985a: 25, 47, 103; 1996: 37-38; Ong, 1997: 81 y ss., 117 y ss.; Toffler, 1993: 452 y ss.; Bell, 1986: 34-36;).

Aquí se llega de nuevo al contacto directo entre el usuario y las competencias técnicas. Los usuarios de un instrumento técnico toman contacto con éste, lo manipulan, lo usan, lo aplican dentro de un contexto social significativo. Este contexto puede ser más o menos general, es decir, puede funcionar más objetivamente, con menores posibilidades de que existan cambios a voluntad para los grupos par- 
ticulares de usuarios, o puede aparecérseles como algo cambiante de acuerdo con sus deseos. Sin embargo, en paralelo con los usos socialmente construidos por los grupos dirigentes y con los usos parasitarios incorporados por los grupos no dirigentes que presentan alternativas, la técnica -como instrumento que persigue un fin- afecta aspectos corporales de los usuarios. Si bien es cierto que el uso técnico siempre se encuentra social y psicológicamente mediado, la relación entre la técnica y el cuerpo se desarrolla de un modo interdependiente con la relación social. Los cambios ocasionados en las capacidades sensitivas, perceptivas, de comportamiento, de memoria y aprendizaje, de la conciencia y de sociabilidad (Bunge, 1988) no están todos determinados directa y únicamente por los aspectos sociales de la técnica. Hay cambios en estas competencias que son universales -por el solo hecho de que un ser humano utilice un instrumento técnico con cierta repetitividad- y otros que responden a diferencias culturales.

Las intuiciones de Mc Luhan y los esquemas ensayados por Walter Ong han sido aplicados, entre otros, por investigadores de la Universidad de Stanford en los años noventa. El estudio más amplio sobre el "entorno" o "ambiente" que desarrollan las tecnologías de la comunicación en contacto con personas sociales en la vida cotidiana lo realizaron Byron Reeves y Clifford Nass (1996). La conclusión más contundente en relación con los efectos técnicos de los medios de comunicación (sobre todo, televisión, ordenadores y sus aplicaciones) es que las personas reaccionan y responden al ambiente técnico de los media de un modo tanto social como natural (Reeves y Clifford, 1996: 251). Los medios se transforman en un entorno inconsciente para los seres humanos. Provocan respuestas emocionales, demandan atención, influyen en la memoria de las personas, modifican ideas y representaciones acerca de lo que es natural o posible. Según las conclusiones de estos investigadores, el entorno mediático influye sobre la personalidad (atributos y relaciones), las emociones, los roles sociales y la percepción de lo que es real y no real. Pero, el aporte más importante de sus investigaciones es haber corroborado que los medios técnicos desarrollan un ambiente al funcionar de un modo sistémico y en conjunto sobre el uso y la selección de las personas que los utilizan. Por otro lado, desde un punto de vista teórico diferente, Donald M. Lowe (1999) alcanza una conclusión que se acerca al argumento que defiende los efectos corporales de los medios. Lowe propone una historia de la percepción de la burguesía, sustentando su hipótesis desde la fenomenología de Merleau-Ponty y desde la teoría de los medios de Walter Ong (Lowe, 1999: 13 y ss.). Según Lowe, los medios alcanzan a jerarquizar el uso de los sentidos. La percepción es una integración y síntesis de los cinco sentidos. Cada medio técnico de comunicación actúa sobre los hábitos sensitivos y perceptivos de los seres humanos.

Las actividades y los fines que la técnica permite alcanzar, se transforman en hábitos o en costumbres que son difícilmente modificables a voluntad por las personas sociales ya que sus efectos funcionan por medio de la naturalización de la vida cotidiana. La "naturalización" de la vida cotidiana es el proceso personal y social, individual y colectivo, privado y público por medio del cual, las personas sociales 
experimentan su vida cotidiana como no separada de ellos, como algo "normal", como una realidad que es natural aunque haya sido producida por cambios históricos y culturales.

Los autores que se declaran "deterministas tecnológicos" -o que son acusados de serlo- no muestran detalles de cómo se podrían dar el tipo de cambios que proponen. A continuación se expondrán algunos de los cambios que se podrían dar en cualquier ser humano si se expone a la interacción con ciertas técnicas de comunicación. Pero, antes, es necesario recordar que estos efectos sólo se desarrollarán cuando se han cumplido, de un modo u otro, las tres fases anteriores: desarrollo de una técnica relativamente eficaz, identificación de problemas por grupos de usuarios y un proceso de estabilización o de institucionalización del uso y de las definiciones. Una vez estabilizada, la técnica activa dos efectos generales: primero, cambia ciertos modos habituales o pautados de las personas que usan la técnica; y segundo, expande y desarrolla un "sistema de sustentación" (Bell, 1989: 317), es decir, una serie de "proveedores" sociales, legales y tecnológicos que cumplen la función de mantener en funcionamiento el uso del artefacto en cuestión.

El primer efecto de los medios de comunicación según los deterministas tecnológicos se realiza sobre los sentidos y sobre la percepción. En realidad, el "sentir" es la capacidad de detectar algo de un modo inmediato (Bunge, 1988: 122). Los medios de comunicación, en tanto técnicas que deberían mejorar las competencias comunicativas, se organizan sobre la manera biológicamente normal de que los seres humanos sienten la realidad. Pero pueden llevar estos umbrales al máximo, es decir, hasta límites que antes no se habían llevado. Segundo, los seres humanos "perciben" cuando interpretan y le dan sentido a lo que sienten. Percibir es reconocer y descifrar un mensaje sensorial en forma de "sucesos", es decir, de relaciones entre cosas determinadas por "mapas" o "esquemas" o "modelos" corporales o externos (Bunge, 1988: 124). Cada medio realiza y tiene menos o más capacidad para jerarquizar o no determinados mapas o modelos perceptivos, es decir, los significados de determinadas posturas corporales, sonidos, formas, colores, etc. Una vez institucionalizado el medio, las capacidades naturales quedan al descubierto. Tercero, los medios actúan sobre las conductas y sobre los motivos. Desde un punto de vista muy básico, la "conducta" debería definirse como un ciclo: (estímulo) (proceso neural) (respuesta) (resultado) (retroalimentación sobre el proceso endocrino) (Bunge, 1988: 136). Los medios actúan en este ciclo. Por un lado, afectando alguno de los momentos de la conducta; por otro, desarrollando una pauta de conducta, es decir, una conducta recurrente. En relación con los motivos, las técnicas de comunicación -como cualquier otra-pueden hacer que los usuarios se sientan o detecten placer o desagrado en el uso. Cuarto, la relación con los medios afecta la memoria y el aprendizaje. Cada uno de los medios de la historia cultural, ha llevado a desarrollar mejor o peor la memoria y el proceso de aprendizaje de los seres humanos que los usan. La diferencia que existe entre tener el control sobre el proceso de almacenamiento y de recuperación del conocimiento o no, es central para el cambio social a largo plazo. Además, el almacenamiento puede ser una copia o una grabación semejante (Bunge, 1988: 150). La diferencia que McLuhan (1996: 43 y ss.) hacía entre medios "fríos" y "calientes" es posible de com- 
prender si se vincula las competencias de cada medio con el tipo de aprendizaje que puede realizar la persona. Y aunque la institucionalización es central para activar o anular el uso de una técnica, una vez activada o anulada, se desarrollan los efectos biológicos. Quinto, los efectos generales se pueden activar en el pensamiento y en el proceso de cognición (Bunge, 1988: 171). El nivel de abstracción de los conceptos adquiridos y de las proposiciones formadas de acuerdo con cada medio, es un efecto del tipo de tarea comunicativa que permite o no cada artefacto $^{2}$.

En definitiva, la creación de un ambiente perceptivo, tal como lo definía casi metafóricamente McLuhan, sólo es posible después de que se compruebe que la técnica resuelve un problema, segundo, de que los modos de uso, los objetivos precisados por los inventores o realizadores, sean aceptados por ciertos grupos o rechazado por otros; tercero, que los usos aceptados y rechazados se transformen en patrones, en pautas de comportamiento hasta llegar a ser inconscientes. Una vez en esta etapa, entonces, es posible pensar en que la técnica en cuestión puede modificar u organizar las capacidades sensitivas, perceptivas, de conducta, de memoria, de aprendizaje y de relaciones sociales.

\section{Aplicación de la teoría multidimensional a casos históricos}

A continuación se expondrán dos casos de tecnologías de la comunicación y de la información. El objetivo es aplicar a cada una de estos instrumentos técnicos el análisis de las proposiciones de la hipótesis expuesta en el apartado 2 y desarrollada en el apartado 3. El criterio de selección de los casos fue el hecho de que cada uno de los medios se inició bajo el marco de referencia de un tipo diferente de conocimiento. La imprenta y el libro fueron el resultado de un conocimiento artesanal y no científico, desarrollado por los gremios y los grupos de maestros artesanos. Por otro lado, la televisión es un medio producto de la ciencia, resultado del descubrimiento de la electricidad. Esta diferencia ha sido marcada por algunos estudiosos del problema de la relación entre técnica y sociedad. Norbert Elias (1995: 43 y ss.; 1990: 61 y ss.), como sociólogo del conocimiento, afirma que la única manera de comprender y de explicar la producción del conocimiento humano es entrelazar el conocimiento no científico con el científico. Mario Bunge (1990: 28), distanciado de Elias, sin embargo, coincide en que la técnica es el resultado de la ciencia, pero que en las sociedades no científicas, era el modo artesanal y tradicional de conocimiento el que producía los artefactos técnicos. Por lo tanto, se utilizarán como casos para aplicar la hipótesis, una técnica nacida del conocimiento no científico y otra nacida de los laboratorios y de la investigación realizada con el procedimiento científico.

\footnotetext{
${ }^{2}$ Se podría continuar con otros niveles de realidad sobre los que la tecnología desarrolla sus efectos, pero se ha llegado hasta el desarrollo de la personalidad y de las relaciones sociales. En estos dos niveles, el psíquico y el social, el efecto de los medios es evidente y no es necesario mostrar sus posibilidades.
} 


\subsection{La imprenta: del conocimiento precientífico a la ciencia y de la ciencia a las masas de lectores}

La imprenta es una técnica que se utilizó para aumentar la velocidad de la edición de textos escritos. Desde el punto de vista interno, la imprenta es un mejoramiento del rendimiento de la escritura y, sobre todo, de la capacidad de edición cuantitativa. Pero el aumento de la velocidad en la edición de un libro no fue el único cambio producido ni tampoco fue la única motivación de sus primeros usuarios. Para el historiador Daniel J. Boorstin (1986: 467, II) la imprenta fue lo que terminó por sustituir a la memoria natural como un medio de almacenamiento y de recuperación de conocimientos. Las diferentes formas de escritura, en realidad, ya lo intentaban. Pero hasta que no fue perfeccionado el proceso técnico de escribir con tinta sobre papel, el método de la memorización era un proceso técnico que se estudiaba y se perfeccionaba. El perfeccionamiento de la técnica de la producción y reproducción de libros era, entonces, lo que estudiosos y bucrócratas estarían buscando para terminar con sus problemas de organización de los datos, cifras y escritos.

De acuerdo con la teoría de la construcción social de la realidad (Pinch y Bijker, 1984; Bikjer, 1993) la edición de libros por medio de la imprenta fue institucionalizado de dos modos diferentes en Oriente y en Occidente. En China y Corea, se había descubierto, antes que en Europa la impresión con tipos móviles (Boorstin, 1986: 486, II). La escasez de maderas duras para fabricar los tipos móviles en Corea, llevó a los impresores a que probasen con metales. Adaptaron las técnicas de la fabricación de monedas a la de la impresión y sancionaron leyes para regular la calidad del proceso de impresión: se castigaba con treinta azotes al impresor que hiciese su trabajo demasiado oscuro o demasiado claro (Boorstin, 1986: 488, II). Luego, en el siglo XVI, y en el proceso de evangelización que los jesuitas pretendían llevar adelante en el Japón, convencieron al daimío de Kyushu que enviara un delegación a visitar al Papa Gregorio XIII; la delegación japonesa regresó con una imprenta y un grupo de expertos europeos. En los tres países, había dificultades con la imprenta de tipos móviles por la escritura ideográfica. A raíz de esto, en Japón, se dejó de utilizar la imprenta después de haber desarrollado un público amplio para el libro impreso, por cuestiones de economía y de practicidad: era demasiado costoso continuar tallando y fundiendo piezas de metal en lengua japonesa. "La impresión tradicional mediante bloques de madera resultada más barata y también más sencilla” (Boorstin, 1986: 491, II).

Por otro lado, los musulmanes tenían otro acercamiento completamente diferente a la imprenta. Al ser el árabe una lengua alfabética (veintiocho letras) era mucho más fácil para la transcripción que los ideogramas chinos, por ejemplo. Pero el Islam rechazó a la imprenta como invento (Boorstin, 1986: 518, II). Y esto tiene sus razones culturales. Desde el momento en que Mahoma (570-632) fue inspirado por revelación divina a escribir el Corán, el árabe se transformó en el idioma oficial y clásico del Islam. La destrucción de la bibioteca de Alejandría se basó en el siguiente razonamiento: si los libros que están en la biblioteca dicen lo mismo y apoyan el Corán, entonces, no se necesitan; si son diferentes y lo contradicen, tampoco se necesitan (Boorstin, 1986: 519, II). Cualquier copia o imitación del estilo del 
Corán se considera un sacrilegio. El primer deber de cualquier niño musulmán era y es aprender de memoria, para recitar, pasajes del Corán. Según Boorstin, el Islam sigue siendo una cultura de la memoria, cultura que no ha podido incorporar a la imprenta y a sus ventajas. La primera impresión del Corán fue realizada por cristianos. Un siglo después de la Biblia de Gutenberg, en 1530, se publicó en Venecia (Boorstin, 1986: 521, II). En resumen, hasta el siglo XVIII y sólo con el imperio otomano, la imprenta fue admitida, con permiso imperial, para imprimir libros dentro de la cultura islámica. Se debía contar con un permiso religioso para componer letras y palabras, pero el muftí prohibió expresamente la impresión del Corán.

En Occidente, la situación se desarrolló de un modo muy diferente. Según Boorstin, la impresión -fabricación de imágenes por contacto-existía en Europa mucho antes de que Johann Gutenberg (13941468) comenzara con su trabajo de impresor (Boorstin, 1986: 491, II). El trabajo de Gutenberg fue la culminación del de muchos otros. Se podría decir, en realidad, que la imagen que Boorstin presenta del trabajo de Gutenberg, las consecuencias más importantes de su trabajo fueron dos: primero, la síntesis de los conocimientos necesarios y suficientes para imprimir; segundo, el proceso de resolución de problemas y de institucionalización, pasando por muchos litigios judiciales y entrando y saliendo de problemas de financiación que fueron antecedentes del uso actual.

En el proceso de invención y de mejoramiento de la imprenta, Gutenberg utilizó sus conocimientos de orfebre y de moldeador de metales (Boorstin, 1986: 492, II). En realidad, su aporte fue al proceso de fabricar tipos móviles de metal. La dificultad que encontraba el impresor de tipos móviles de metal era que debía producir tipos de metal del mismo tamaño y debían poder ser intercambiables. Gutenberg fabricó o inventó una máquina herramienta: una productora de tipos móviles (Boorstin, 1986: 492, II). Pero además, realizó una serie de adaptaciones y sintentizó el conocimiento de los orfebres, de los impresores y de los encuadernadores.

Una vez desarrollada y probada la eficacia de la técnica aparecieron los diferentes grupos sociales relevantes para la aplicación y uso de la impresión (Pinch y Bijker, 1984, Bijker, 1993). De acuerdo con Boorstin, en los primeros años de la imprenta en Europa, había varios grupos sociales que se disputaban la definición de la producción de libros: los copistas, los impresores, los bibliófilos. Los copistas defendían su trabajo y la definición de libro era la de un objeto lujoso y tradicional. Los impresores, en cambio, pensaban y actuaban de un modo más parecido a empresarios. Debían arriesgarse cada vez que imprimían un libro. En tercer lugar, los compradores y coleccionistas de libros seguían valorando los atributos del libro manuscrito, aunque compraban y guardaban los nuevos libros impresos. Algunos impresores, usaban la nueva técnica de copiar con la imprenta, pero luego, con borrador y pincel le daban apariencia de libro manuscrito (Boorstin, 1986: 497, II). Los profesores de las universidades, los vendedores y los bibliotecarios eran otros tres grupos que tenían conflictos de intereses. Los vendedores de libros no eran controlados, con lo cual, no era seguro el origen y la autenticidad de los libros que comercializaban. Los 
vendedores comercializaban obras prohibidas, traducciones proscritas y textos no autorizados. Para combatir la falta de control de los vendedores, los profesores tenía la obligación de dejar sus conferencias y sus clases manuscritas en los "estacionarios"3 de las universidades, que además, cumplían el papel de hacer préstamos de la biblioteca y autorizar los textos (Boorstin, 1986: 513, II).

Otros grupos que interactuaban con el libro manuscrito e impreso fueron los estudiantes de bajos recursos económicos, los bibliotecarios y las personas ciegas (Boorstin, 1986: 515-516, II). Para los "estudiantes pobres", la producción de libros por medio de la imprenta les permitió adquirirlos a bajos precio y leerlos en las bibliotecas o estacionarios. En segundo lugar, las bibliotecas comenzaron a ser lugares para resguardar y mejorar las condiciones físicas de los lectores. Por lo tanto, dejaron de ser meros "estacionarios" o depósitos de libros para ser un lugar de consulta y trabajo de lectura. Y los bibliotecarios pasaron de ser empleados mal pagos y sin preparación específica a ser estudiosos y especialistas en diferentes temas tratados en los libros de la biblioteca. Finalmente, tres generaciones después del invento de Gutenberg, se le dio paso a los invidentes a la lectura de libros: por un lado, Valentin Haüy (1745-1822) "diseñó un tipo itálico simplificado de letra en relieve (Boorstin, 1986: 517, II); por otro, en 1829, Louis Braille (1809-1852) presentó un manual sobre la base de caracteres romanos. En definitiva, había grupos sociales que tenían intereses diferentes en relación con el uso de la imprenta, pero también otros grupos que sólo se diferencian por sus experiencias: las nuevas generaciones aceptarán el libro impreso y se olvidarán del manuscrito. Pero nadie en el año 1488 podía predecir cómo evolucionaría el uso de la técnica en el futuro. El uso dado por cada uno de los grupos, la importancia de estos grupos en la sociedad y sus acciones para influir sobre la institucionalización definitiva del libro son los mecanismos sociales que afectan a la técnica de producción del libro y éste como medio técnico de comunicación y de almacenamiento y recuperación de conocimientos. Por otro lado, las condiciones políticas y culturales de Europa permitieron la existencia del grupo de los "vendedores", que gracias a la ausencia de un control demasiado despótico (como el que había en Asia o en Medio Oriente) podían introducir toda clase de libros, incluyendo aquellos que serían desechados por falta de autenticidad. En el año 1500, estas condiciones político-culturales de base, se suman a la producción de libros como nunca antes se había conocido en Europa (los cálculos más bajos son 10 millones de libros para una población de 100 millones de personas) (Boorstin, 1986: 513, II). Y ambas condiciones, permite un intercambio de ideas y de conocimientos nuevos que se convertirán en los antecedentes de un escenario público cultural mucho más amplio y libre que en Oriente.

Estos cambios están dentro del concepto de "sistema de sustentación” que Daniel Bell (1989: 317) utiliza para explicar el concepto de "productividad". El cambio que produce una nueva técnica sobre

${ }^{3}$ Dice Boorstin, que se denominaba "estacionario" porque, a diferencia de los vendedores ambulantes, permanecía en un lugar: la biblioteca de la universidad, por ejemplo (Boorstin, 1986:512-13, II). 
las medidas de productividad en un área específica de vida social, se derivan, no sólo de la técnica sino del modo en que se organiza la actividad sobre criterios nuevos, modificados por la tecnología en cuestión. Algo similar afirmaba Marshall McLuhan al definir "medio"; según él, una técnica en particular (el automóvil) no era el medio, sino que, en realidad, el medio se formaba por el conjunto de derivaciones necesarias para mantenerlo en funcionamiento y que influían sobre las personas (talleres mecánicos, carreteras, gomerías, autopistas, semáforos, policías de tránsito, señales, calles, etc.). En cuanto al libro, las bibliotecas, la formación de los bibliotecarios, las condiciones físicas de las bibliotecas son otras técnicas que sustentan o sostienen al libro y a la imprenta como medios de adquisición de conocimiento, de memorización y de recuperación del datos. Y es el conjunto lo que forma el ambiente inconsciente para la mayoría de los usuarios.

Otro efecto importante de la imprenta fue el impulso que realizó sobre el uso de las lenguas habladas en Europa. Los impresores necesitaban ampliar al máximo sus públicos de lectores, por lo tanto, usaron las lenguas romances y germanas habladas en las diferentes aldeas europeas (Boorstin, 1986: 498 y ss., II). Según George Duby (1997: 68), la difusión de la imprenta en Europa, además, estuvo relacionado con el desarrollo y expansión del Humanismo en el siglo XIV. Desde el Rin se extiende a toda Europa occidental. Junto con el Humanismo también se desarrolla la Reforma. Los nacionalismos sustentados en las lenguas, el Humanismo de Petrarca o de Erasmo y la Reforma luterana utilizaron la imprenta y fueron, al mismo tiempo, el resultado de ella ya que les permitió el aumento en la velocidad de producción y la identificación con una nueva tecnología que representaba el cambio y la modernización. En resumen, el proceso de prueba y de aplicación del artefacto técnico, seguido por el proceso de problematización con el contacto de diferentes grupos sociales, termina en un proceso de institucionalización que define y produce las condiciones para que comiencen a producirse los efectos técnicos y universales de la técnica sobre el cuerpo o el sistema nervioso del ser humano. En el caso del sistema de impresión, Ong (1997: 117-136) considera que modificó la capacidad de percepción del espacio y reestructuró la manera de experimentarlo.

\subsection{El televisor: ciencia y entretenimiento}

En 1896, el físico alemán Ferdinand Braun llegó a la conclusión de que su campo de estudio (la radiación en tubos al vacío) estaba "sobreexplotado" (Burke, 1998: 48-49). Entonces, decidió orientar su investigación hacia los rayos catódicos en sí. Construyó un tubo al vacío con un cuello que se abría a una pantalla fosforescente. Con esto, corroboró lo que Heinrich Hertz había demostrado pero nadie hasta el momento había podido observar de modo directo: que la electricidad tenía ciclos positivos y negativos. Los cambios en la corriente, de negativa a positiva, y viceversa, permitió movilizar un haz de luz en la pantalla fosforescente. El invento se llamó "osciloscopio" y era muy práctico para medir y observar las características de cualquier corriente eléctrica. Pero además, fue el "precursor del televisor moderno" (Burke, 1998: 49). Tal como es posible entender en el relato de James Burke, la televisión tuvo sus 
antecedentes en conocimientos altamente especializados producidos por científicos. El cambio con respecto al libro y a la imprenta es evidente: sólo con conocimientos con que contaban los artesanos, prácticos pero no científicos, fue posible el comienzo de un medio tan decisivo como la imprenta. Pero en el marco de una sociedad científica, los descubrimientos e inventos se sustentan en el conocimiento producido bajo el procedimiento del método científico. Y la prueba de esto es que, casi al mismo tiempo del trabajo de Braun, otro científico de la misma nacionalidad, Paul Nipkow, en 1884, estaba experimentando con un "disco explorador de imágenes con la intención de convertir las fotografías en señales que pudieran enviarse por cable” (Burke, 1998: 59). Si bien, Nipkow fracasó, esta técnica se utilizaría en los comienzos de la televisión hasta el desarrollo del dispositivo explorador electrónico. En resumen, desde el punto de vista internalista, la televisión es un artefacto que permite ver imágenes en movimiento con el agregado de sonido, que están ocurriendo a una distancia que no es posible de ser sentida por los órganos humanos. Este artefacto es producto de otros inventos: el transistor, el tríodo (detector de ondas hertzianas y amplificador que emite electrones hacia una placa); luego al circuito integrado, en una segunda etapa, aumentando la velocidad de la transmisión y bajando el costo de energía (Flichy, 1993: 184).

En las primeras décadas del siglo XX, una vez asegurados los principios técnicos de la electricidad aplicada a la producción de imágenes y a su transmisión, los avances en el desarrollo técnico de la televisión como artefacto de transmisión de imagen y sonido, orientaban a la técnica hacia un uso experimental, artístico y de cultura académica. En definitiva, se pretendía un medio experimental. Quienes conocían los aspectos técnicos del nuevo medio, consideraban que representaría un avance para acercar la cultura académica o de elite a quienes no podían tenerla de un modo directo. Desde el punto de vista experimental, la televisión podía ser un medio estético extremadamente innovador ya que seguía el modelo de la radio pero con el agregado de imágenes en movimiento, superaba al cine en su capacidad de transmisión de las representaciones ya que éste reduce las posibilidades espacio temporales para transmitir el texto audiovisual, y superaba a la radio porque la representación está compuesta de más de una sustancia expresiva, siendo hasta el momento casi impensable que se pudiese estar mirando lo que alguien hacía a muchos kilómetros de distancia pero en tiempos sincronizados. La construcción social de la televisión fue y es un proceso social que se sustentó en las experiencias de la organización de la radio. Los conflictos identificados por Pinch y Bijker entre los grupos sociales relevantes para el medio, se produjeron, en primer lugar, entre los aficionados y técnicos que pretendían orientar a la televisión como un medio experimental, de arte y de conocimientos serios, y los empresarios que pretendían hacer del medio un negocio. La televisión es el resultado, también, del arreglo entre los aficionados, los técnicos y los empresarios: hacen una alianza para que el medio sea una posibilidad real de uso. En la Argentina de los años cincuenta, por ejemplo, el negocio que identificaron en los inicios los empresarios de la radiodifusión fue el de la importación y venta de aparatos de televisión (Ulanosvky, 1976: 14). El interés por los contenidos estaba en un segundo plano al principio del proceso de tecni- 
ficación mediática. La prueba de esto es que fue Jaime Yankelevich el empresario que compró los primeros equipos de televisión en los Estados Unidos: su negocio era el de la radio, pero fundamentalmente, el de los artefactos eléctricos y repuestos para instalaciones electrónicas (Ulanovsky, 1976: 13). Las intenciones de los aficionados y de los experimentadores de la televisión no fueron cumplidas, ya que el contenido de la televisión se tomó de la radio y un poco del cine. Luego vendrían el circo, el ballet y el fútbol: nada de todo esto se había pensado en la televisión experimental de los aficionados e inventores.

Desde el punto de vista del proceso de estabilización (Pinch y Bijker, 1984: 416) o de institucionalización (Williams, 1975: 12-13, 1982: 31-52), la televisión fue organizada como la radio, bajo diferentes reglas: del mercado de la publicidad, del Estado, de la sociedad civil. Los diferentes grupos dirigentes, en distintos países o en diferentes momentos históricos, definieron o intentaron definir a la televisión de manera que coincida con sus intereses políticos, económicos o ideológicos. Cada sociedad, soluciona la administración de la televisión de un modo diferente, dice Williams (1975: 34). Hay Estados que son sólo garantías sobre los contenidos, otros que se despreocupan completamente y unos terceros que los controlan de un modo muy estricto y detallado, por ejemplo, la ex Unión Soviética.

Finalmente, el proceso de masa crítica y de difusión de la televisión, desarrollaron las condiciones para que los seres humanos (Reina Schement y Curtis, 1995: 108-113; Elizalde, 2002: 388 y ss; Williams, 1975) comenzaran a modificar ciertos procesos corporales, psíquicos y sociales de un modo general. En primer lugar, la televisión tiene la capacidad de afectar los sentidos y la percepción en tanto muestra un espectro visual que puede ser extraño para la vida no mediática de las personas. En segundo lugar, condiciona a pensar de un modo menos abstracto: la imagen televisiva permite la realización de otras actividades mientras el espectador se expone a la pantalla. Este efecto de percepción social es el resultado de la institucionalización. Parecería que Raymond Williams tiene razón en este punto. El formato estético de la televisión depende de reglas sociales y no sólo de aspectos técnicos. Con géneros televisivos más abstractos, sería imposible un espectador desdoblado en dos actividades mientras mira un programa de televisión. Tercero, la televisión tiene varias capacidades comunicativas únicas según De Kerckhove (2002: 2-3): coordina las emociones en grupos sociales espacio temporalmente distantes, construye objetos públicos y genera una comunidad entre personas que no se comunican directamente entre sí, sino de un modo virtual. Estas ideas son compatibles con el pensamiento de Paolo Vidali (1995: 263 y ss.) ya que compara a la televisión con los llamados "nuevos medios" (medios no tradicionales o no masivos). Según Vidali, la televisión fue un medio que modificó la experiencia de lo cotidiano. La forma que ha adquirido la televisión es de ambiente o entorno en sí misma. La televisión ha modificado los criterios de sentir el espacio y el tiempo, la percepción de lo normal y lo anormal, ya que es muy eficaz para transformarse en ambiente. El punto de vista del constructivismo social del psicólogo Kenneth Gergen (1992: 82 y ss.) va mucho más allá. Según Gergen, los medios audiovisuales, y especialmente la televisión, ha desarrollado nuevas formas de relaciones interpersonales: es posible tener y mantener relaciones significativas con personas que no saben que existen esas relaciones existencialmente significativas. Otra 
consecuencia en la misma dirección, dice Gergen (1992: 86 y 94) es la proliferación de relaciones sociales: tanto en cantidad como en variedad. La tesis de un grupo de investigadores en el que participa Gergen considera, además, que la televisión ha colaborado en el desarrollo del "yo" (self) de un modo especial (Carbaugh, 1996: 84 y ss.; Priest, 1996: 77 y ss.). En los talk show las personas negocian su estructura del "yo" (Priest, 1996: 68-69) mientras que la organización institucional aporta y se complementa con las competencias comunicativas del medio como lo muestra Donal Carbaugh (1996: 89) en el contexto de los receptores en la Unión Soviética.

\section{Conclusiones}

Como se anunció, el objetivo del trabajo era reunir los argumentos que explican el proceso completo de relación entre la tecnología y la sociedad, sin caer en el error de la "discusión entre sordos". Esa es la sensación después de repasar los argumentos y compararlos con los casos históricos: que las diferentes posiciones teóricas no se han escuchado lo suficiente como para compaginar una teoría que considere, no los efectos más importantes, sino los diferentes efectos y determinaciones entre la sociedad y la tecnología.

En primer lugar, la técnica afecta el mundo social, psicológico y cultural gracias a que modifica los modos de hacer con más eficacia ciertas actividades. Afecta la relación social de resolución de tareas, concede cierto poder social a un grupo social que tiene el monopolio del conocimiento para aplicar la técnica. Si una técnica determinada permite resolver de un modo más rápido el proceso de escritura, es posible que poco a poco se extiendan cambios en el modo de escribir y en otras actividades sociales que antes eran afectadas por la mayor dificultad para escribir.

Segundo, el proceso de imaginación, desarrollo, planificación, aplicación y adopción de una técnica no puede ser explicado por ninguna de las hipótesis de un modo independiente. Es necesario integrar los argumentos que no son contradictorios para alcanzar una explicación más compleja de un fenómeno complejo.

Tercero, en la discusión se pone en juego el concepto de "naturaleza humana". Desde este punto de vista, en un extremo se encuentran quienes tienen una concepción fuerte de ésta, de tal modo que creen que los aspectos comunes son más cantidad y están más a la superficie que las diferencias culturales y sociales. Del otro lado, quienes tienen una concepción más débil de la naturaleza humana, o menos innatista, creen que las diferencias son más importantes que los puntos en común.

En cuarto lugar, el desarrollo de una técnica es lo suficientemente complejo como para derivar en efectos impensados por sus inventores. Los casos analizados lo muestran. La imprenta se construye y piensa gracias a un conocimiento pre científico y artesanal, y termina afectando la producción de conocimiento científico. Mientras que, la televisión, al contrario, es el resultado del conocimiento científico en una primera etapa, pero modifica la vida cotidiana y el conocimiento de sentido común. 
Finalmente, en los diferentes argumentos de la discusión aparece un problema que sólo se ha tratado de modo implícito. Lo que está en juego en el debate, además, es la amplitud o limitación de la definición de "técnica". Y esto deberá discutirse en función de sus consecuencias políticas, económicas y sociales en general.

En definitiva, para explicar el modo en que la sociedad -el mundo social histórico y cultural- determina cambios sobre los instrumentos técnicos y de qué manera las técnicas cambian patrones de comportamiento, de pensamiento, de emociones, de representaciones de la realidad social es necesario contar con una teoría que integre los argumentos de teorías que normalmente están enfrentadas entre sí. El descubrimiento de un cambio en una técnica ya utilizada o la invención de una nueva, está motivada y organizada por el cumplimiento eficaz de un objetivo o fin. Luego, la posibilidad de que se aplique, se desarrolle y se adopte, depende de los procesos de institucionalización y de aceptación más o menos crítica de esta institucionalización. El problema es que los sociólogos de la técnica o de la ciencia no sólo creen que esta institucionalización es un agregado que la sociedad hace a la técnica o al conocimiento científico, sino que es una parte inherente a éstos. Las institucionalizaciones y los procesos de reconstrucción social de la técnica, resultado del encuentro que los grupos con mayor o menor poder político, cultural y económico, afecta a la técnica y lleva a cambios en los mecanismos estrictamente técnicos, agregados o transformaciones que se adapten más a las necesidades sociales de los usuarios. Finalmente, los procesos de modificación social de la tecnología no detienen los efectos directos de ésta sobre las personas, los grupos y las organizaciones sociales.

\section{Referencias bibliográficas}

- Bell, D. (1986) "La telecomunicación y el cambio social”, en M. de Moragas (ed,) Sociología de la comunicación de masas. IV. Nuevos problemas y transformación tecnológica. México: Gustavo Gili, pp. 34-55.

- Bell, D. (1989) El advenimiento de la sociedad postindustrial. Un intento de prognosis social. Madrid: Alianza.

- Bettetini, G. (1995). “Tecnología y comunicación”, en Bettetini, G. y Colombo, F. Las nuevas tecnologías de la comunicación. Barcelona: Paidós, pp. 15-39.

- Berger, P. Luckmann, Th., (1989) La construcción social de la realidad. Buenos Aires: Amorrortu editores.

- Bijker, W.E. (1993) "Do Not Despair: There is Life after Constructivism”, Science, Technology,\& Human Values, Vol. 18, N. 1, Theme Issue: Technological Choices (Winter), pp. 113-138.

- Boorstin, D.J. (1986) Los descubridores. Volumen II: la naturaleza y la sociedad. Barcelona: GrijalboMondadori. 
- Bunge, M. (1980) Epistemología. México: Siglo veintiuno editores.

- Bunge, M. (1988) El problema mente-cerebro. Un enfoque psicobiológico. Madrid: Tecnos.

- Bunge, M. (1991) “El impacto social de la innovación tecnológica”, en Drago T. y Ruíz de Gopegui, L. Innovación tecnológica y comunicación, Mérida, V Encuentro Ibeoramericano de Comunicación, pp. 26-41.

- Bunge, M. (1998) Sociología de la ciencia. Buenos Aires: Editorial Sudamericana.

- Burke, J. (1998) El efecto carambola. Barcelona: Planeta.

- Carbaugh, D. (1996) "Mediating Cultural Selves: Soviet an American Cultures in Televised 'Spacebrigde”, in D. Grodin and Th. Lindlof, Constructing The Self in a mediated World. Inquieris in social construction. Thonsand Oaks: Sage Publications, pp. 84-106.

- De Kerhove, D. (2002) “The Internet Enters Television, a Trojan Horse in The Public Mind", http:// www.mcluhan,utoronto.ca/article_internettelevision.htm, The McLuhan Program in Culture and Technology, 7 pp.

- Douglas, M. (1993) Cómo piensan las instituciones. Madrid: Alianza Editorial.

- Duby, G. (1997) Atlas Histórico Mundial. Madrid: Editorial Debate.

- Elias, N. (1990) "Los pescadores en el Maëltrom”, en N. Elias: Compromiso y distanciamiento. Ensayos de sociología del conocimiento. Barcelona: Ediciones Península, pp. 61-152.

- Elias, N. (1995) Sociología fundamental. Barcelona: Gedisa.

- Elias, N. (1998) “Tecnificación y civilización”, en La civilización de los padres y otros ensayos. Santa Fe de Bogotá: Editorial Norma, pp. 451-507.

- Elizalde, L. (2002) "Radio y televisión”, en Nueva Historia de la Nación Argentina, tomo 9: "La Argentina del siglo XX”, Buenos Aires: Editorial Planeta, pp. 363-394.

- Ellul, J. (1964) The technological society. New York: Vintage Books.

- Flichy, P. (1993) Una historia de la comunicación moderna. Espacio público y vida privada. México: Gustavo Gili.

- Gehlen, A. (1980) Hombre. Su naturaleza y su lugar en el mundo. Salamanca: Ediciones Sígueme.

- Gergen, K. (1992) El yo saturado. Dilemas de identidad en el mundo contemporáneo. Barcelona: Paidós.

- Giddens, A. (1997) Sociología. Madrid: Alianza Editorial. 
- Habermas, J. (1989) “Ciencia y técnica como 'ideología”, en Ciencia y técnica como ideología. Madrid: Tecnos, pp. 53-112.

- Hirsch, R. (1990) “Imprenta y lectura entre 1450 y 1550”, en A. Petrucci (comp.) Libros, editores y público en la Europa moderna. Valencia: Edicions Alfons el Magnánim, Institució Valenciana D’Estudis i Investigació, pp. 27-70.

- Katz, E. y Dayan, D. (1995) La historia en directo. La retransmisión televisiva de los acontecimientos. Barcelona: Gustavo Gili.

- Lowe, D.M. (1999) Historia de la percepción burguesa. Buenos Aires: Fondo de Cultura Económica.

- Luhmann, N. De Georgi, R. (1993) Teoría de la sociedad. México: Universidad de Guadalajara, Universidad Iberoamericana, Instituto Tecnológico de Estudios Superiores de Occidente.

- McLuhan, Marshall. (1960) "The effect of the printed book on language in the $16^{\text {th }}$ Century", in Carpenter, E. y McLuhan, M. Explorations in Communication. Boston: Beacon Press, pp. 125-135.

- McLuhan, M. (1971) Contraexplosión, Buenos Aires, Ed. Paidós.

- McLuhan, M. (1985a) La galaxia Gutenberg. Génesis del Homo Typographicus. Barcelona: Planeta-Agostini.

- McLuhan, M. y Q. Fiore (1985b) Guerra y paz en la aldea global. Barcelona: Planeta-Agostini.

- MacLuhan, M. (1996) Comprender los medios de comunicación. Las extensiones del ser humano. Barcelona: Paidós.

- McQuail, D. (1993) Introducción a la teoría de la comunicación de masas. Barcelona: Paidós (segunda edición).

- Merton, R. (1992) “La máquina, el trabajador y el ingeniero”, en Teoría y estructura social.México: Fondo de Cultura Económica, pp. 648-659.

- Oliver, P.E. Marwell, G. (2001) "Whatever Happened to Critical Mass Theory? A Retrospective and Assessment”, Sociological Theory, Vol. 19, N. 3, (Nov.), pp. 292-311.

- Ong, W.J. (1967a) The presence of the Word. Some Prolegoma of Culture and Religious History. New Haven: Yale University Press.

- Ong, W. (1967b). "Breakthrough in Communications", in In the human grain. Further explorations of contemporary culture. The Macmillan Company: New York, p. 1-16.

- Ong, W.J. (1977) Interfaces of the Word. Studies in the Evolution of Consciousness and Culture. Ithaca: Cornell University Press. 
- Ong, W. J. (1997) Oralidad y escritura. Tecnologías de la palabra. México: Fondo de Cultura.

- Pinch, T.J. Bijker, W.E. (1984) "The Social Construction of Facts and Artefacts: Or How the Sociology of Science and the Sociology of Technology Might Benefit Each Other”, Social Studies of Science, Vol. 14, N. 3, pp. 399-441.

- Priest, P. (1996) “' 'Gilt by Association': Talk Show Participants' Televisually”, in D. Grodin and Th. Lindlof, Constructing The Self in a mediated World. Inquieris in social construction. Thonsand Oaks: Sage Publications, pp. 68-93

- Reina Schement, J. y Curtis, T. (1995) Tendencies and Tensions of the Information Age. The Production and Distribution of Information in the United States. New Brunswikc: Transaction Publishers.

- Reeves, B. and Nass, C. (1996) The Media Equation. How People Treat Computers, Television, and New Media Like Real People and Places. New York: Center for Study og Language and Information, Cambridge University.

- Sartori, G. (1992) “Videpoder”, en G. Sartori Elementos de teoría política. Buenos Aires: Alianza Editorial, pp. 305-316.

- Schelling, Th. (1978) Micromotives and Macrobehavior. New York London: W.W. Norton \& Company.

- Schelsky, H. (1967a) "El hombre en la civilización científica”, en El hombre en la civilización científica y otros ensayos. Buenos Aires: Sur, pp. 7-42.

- Schelsky, H. (1967b) “Acerca de la estabilidad de las instituciones, en especial de las constituciones. (Reflexiones antropológico-culturales sobre un tema jurídico-sociológico)", en El hombre en la civilización científica y otros ensayos. Buenos Aires: Sur, pp. 43-66.

- Searle, J. (1997) La construcción de la realidad social. Paidós, Barcelona, (edición en inglés, 1995).

- Toffler, A. (1993) El shock del futuro. Barcelona: Plaza \& Janes Editores.

- Ulanovsky, C. (1976) 1951-1976 Televisión argentina 25 años después. Buenos Aires: Hachette.

- Vidali, P. (1995) “Experiencia y comunicació en los nuevos media”, en G. Bettetini y F. Colombo Las nuevas tecnologías de la comunicación. Barcelona: Paidós, pp. 259-286.

- Weber, M. (1996) Economía y sociedad. Esbozo de sociología comprensiva. México: Fondo de Cultura Económica.

- Williams, F., Strover, Sh., Grant, A.E. (1996) "Aspectos sociales de las tecnologías de los nuevos medios", en Bryant, J. y Zillman, D. (comps): Los efectos de los medios de comunicación. Investigaciones y teorías. Barcelona: Paidós, pp. 617-642. 
- Williams, R. (1971) Los medios de comunicación social. Barcelona: Ediciones Península.

- Williams, R. (1975) Television. Technology and Culture Form. New York: Schoken Books.

- Williams, R. (1982) Cultura. Sociología de la comunicación y del arte. Barcelona: Paidós.

- Williams, R. (1984) “Cultura y tecnología”, en Hacia el año 2000, Barcelona: Grijalbo, p. 150-177.

- Williams, R. (1989) “Cultura y tecnología”, en La política del Modernismo.Contra los nuevos conformistas. Buenos Aires: Manantial, pp. 151-174. 